\title{
An Efficient Privacy Preserving Keyword Search Scheme in Cloud Computing
}

\author{
Qin Liu ${ }^{\dagger}$, Guojun Wang ${ }^{\dagger \dagger *}$, and $\mathrm{Jie} \mathrm{Wu}^{\ddagger}$ \\ ${ }^{\dagger}$ School of Information Science and Engineering \\ Central South University \\ Changsha 410083, Hunan Province, P. R. China \\ * Correspondence to: csgjwang@ @ail.csu.edu.cn \\ $\ddagger$ Department of Computer Science and Engineering \\ Florida Atlantic University \\ Boca Raton, FL 33431, USA
}

\begin{abstract}
A user stores his personal files in a cloud, and retrieves them wherever and whenever he wants. For the sake of protecting the user data privacy and the user queries privacy, a user should store his personal files in an encrypted form in a cloud, and then sends queries in the form of encrypted keywords. However, a simple encryption scheme may not work well when a user wants to retrieve only files containing certain keywords using a thin client. First, the user needs to encrypt and decrypt files frequently, which depletes too much CPU capability and memory power of the client. Second, the service provider couldn't determine which files contain keywords specified by a user if the encryption is not searchable. Therefore, it can only return back all the encrypted files. A thin client generally has limited bandwidth, CPU and memory, and this may not be a feasible solution under the circumstances. In this paper, we investigate the characteristics of cloud computing and propose an efficient privacy preserving keyword search scheme in cloud computing. It allows a service provider to participate in partial decipherment to reduce a client's computational overhead, and enables the service provider to search the keywords on encrypted files to protect the user data privacy and the user queries privacy efficiently. By proof, our scheme is semantically secure.
\end{abstract}

Keywords: cloud computing; privacy preserving; searchable encryption; partial decipherment

\section{INTRODUCTION}

Cloud computing [1], which dynamically provides reliable services over the Internet, is one of the 2009 Top 10 Strategic Technologies [2]. Recently, many academic and industrial organizations have started investigating and developing technologies and infrastructure for cloud computing. The representative cloud platforms include Amazon Elastic Compute Cloud (EC2) [3], Google App Engine [4], and Microsoft Live Mesh [5].

A user stores his personal files in a cloud, and retrieves them wherever and whenever he wants. We consider the following application: a user $U$ pays a service provider $S$ for a storage service in order to store his email messages, and later he wants to retrieve only emails containing certain keywords when he is traveling with a thin client, such as a wireless PDA and a mobile phone. It is trivial to do so when the email messages are stored in the form of a plain-text, which will introduce undesirable security and privacy risks. For example, $U$ is a technician in Company A who takes in charge of after-sale services. He stores all the emails sent from the customers in a cloud when he is in his office with a desktop, and retrieves them to tackle the customer's service requests when he is out with a PDA. An attacker who intercepts and captures the communications is able to know the customer's privacy information as well as some important business secrets. What is worse is that an untrustworthy service provider is able to easily obtain all the information and sell it to the biggest rival of Company A. As described in Haclgiimfi et al [6], there are two main attacks under such a circumstance, i.e., outer attacks initiated by unauthorized outsiders and inner attacks initiated by untrustworthy service providers. In some cases, we couldn't fully trust a service provider, but still need its service. Therefore, it needs to provide some mechanisms to protect the user data privacy and the user queries privacy in cloud environment.

The simplest solution is to encrypt the emails before storing them in a cloud and send queries in the form of encrypted keywords. For example, a user may use his public key to encrypt the email message body and its keywords before sending it to a service provider, and then sends queries in the form of encrypted keywords to retrieve the email. Since the secret key is only known to the user himself, an attacker has no idea of the encrypted files and the user queries patterns. However, such a simple encryption scheme may introduce other problems: (1) It depletes too much CPU capability and memory power of the client during the encryption and decryption; (2) The service provider couldn't determine which emails contain keywords specified by a user if the encryption is not searchable. Therefore, it can only return back all the encrypted emails. Generally speaking, a thin client has only limited bandwidth, CPU and memory, therefore a simple encryption scheme couldn't work well under these circumstances.

In this paper, we dedicate to solve the above problems and propose an efficient privacy preserving keyword search scheme in cloud computing. Our contributions are threefold:

1) It supports keyword search on encrypted data. It enables a service provider to determine whether a given email contains certain keywords specified by a user, but have no idea of any information about both the specified keywords and the encrypted emails. It is able to protect the user data privacy and the user queries privacy efficiently 
during the search process.

2) It is efficient and practical. It enables a service provider to participate in partial decipherment so as to reduce a user's computational overhead, but has no ability to recover the plain-text. It is well suited to be applied to cloud computing.

3) It is a provably secure scheme. We first define an efficient privacy preserving keyword search scheme, and give its security definition in the sense of semantic security. And then we construct the scheme based on bilinear maps, and prove that it is semantically secure assuming the BDH problem is hard [8].

This paper is structured as follows. We first review some important background and definitions in Section 2. We then describe an efficient privacy preserving keyword search scheme and give its security definition in Section 3. We construct the scheme based on bilinear maps and prove that it is semantically secure in Section 4. Finally we present our future work and conclude this paper in Section 5.

\section{Preliminaries}

\section{A. Related Work}

Boneh et al [7] introduce a public key encryption with keyword search (PEKS) scheme, which supports the keyword search on encrypted data. The application context is as follows: (1) Bob sends to Alice an email encrypted under Alice's public key; (2) Alice's email gateway wants to test whether the email contains the keyword urgent so that it could route the email accordingly; (3) But Alice does not want the email gateway to be able to decrypt her messages. Boneh et al define and construct a mechanism that enables a gateway to test whether the word urgent is a keyword in the email using a trapdoor provided by Alice, but learn nothing about the email. This notion could be applicable to cloud environment with some improvements.

In cloud environment, Alice and Bob might be the same person who pays for a storage service. If a user wants to retrieve emails containing certain keywords when he is traveling with a PDA, PEKS couldn't work well. According to PEKS, a service provider returns back encrypted emails after finishing the search, and a user needs to decrypt them by himself. Frequent decryption will deplete too much CPU capability and memory power of the client and lose critical virtue of cloud computing. For the sake of reducing a user's computational overhead, we allow a service provider to participate in partial decipherment, but keep the plain-text to be blind to the service provider. Under normal circumstances, a user stores encrypted files once, but retrieves and decrypts them for many times. Our approach could reduce a user's computational overhead largely.

Diament et al [11] first introduce the notion of an efficient dual receiver cryptosystem, which enables a ciphertext to be decrypted by two independent receivers. The main disadvantage of the dual receiver cryptosystem is that the server needs to send an auxiliary private key to a client for decrypting a partial ciphertext, which is insecure in the real environment. Recently, there is much work on keyword search on encrypted files, such as Song et al [9], Bennett et al [10], and Chang et al [12]. In this paper, we borrow the idea of partial decipherment, and propose an efficient privacy preserving keyword search scheme by improving PEKS, which requires no private key transmission and is more applicable to a cloud environment.

\section{B. Related Definitions}

In this section, we introduce some definitions in Boneh et al [8] to form the basis of our scheme. Let $\mathbb{G}_{1}$ and $\mathbb{G}_{2}$ be two cyclic groups of some large prime order $q$. We view $\mathbb{G}_{1}$ as an additive group and $\mathbb{G}_{2}$ as a multiplicative group.

Definition 2.1 (Bilinear Maps): We call $e$ a bilinear map if $e: \mathbb{G}_{1} \times \mathbb{G}_{1} \rightarrow \mathbb{G}_{2}$ is a map with the following properties:

1) Computable: There is a polynomial time algorithm to compute $e(g, h) \in \mathbb{G}_{2}$, for any $g, h \in \mathbb{G}_{1}$.

2) Bilinear: $e\left(g^{x}, h^{y}\right)=e(g, h)^{x y}$ for all $g, h \in \mathbb{G}_{1}$ and all $x, y \in \mathbb{Z}_{q}^{*}$.

3) Non-degenerate: if $g$ is a generator of $\mathbb{G}_{1}$, then $e(g, g)$ is a generator of $\mathbb{G}_{2}$.

Definition 2.2 (BDH Parameter Generator): We say that a randomized algorithm $I G$ is a $\mathrm{BDH}$ parameter generator if IG takes a sufficiently large security parameter $K>0$, runs in polynomial time in $K$, and outputs the description of two groups $\mathbb{G}_{1}$ and $\mathbb{G}_{2}$ of the same prime order $q$ and the description of a bilinear map $e: \mathbb{G}_{1} \times \mathbb{G}_{1} \rightarrow \mathbb{G}_{2}$.

Definition 2.3 (BDH Problem): Given a random element $g \in \mathbb{G}_{1}$, as well as $g^{x}, g^{y}$, and $g^{z}$, for some $x, y, z \in \mathbb{Z}_{q}^{*}$, compute $e(g, g)^{x y z} \in \mathbb{G}_{2}$.

Definition 2.4 (BDH Assumption): If $\mathcal{I G}$ is a BDH parameter generator, the advantage $A d v_{I \mathcal{G}}(\mathcal{B})$ that an algorithm $\mathcal{B}$ has in solving the $\mathrm{BDH}$ problem is defined to be the probability that $\mathcal{B}$ outputs $e(g, g)^{x y z}$ on inputs $\mathbb{G}_{1}, \mathbb{G}_{2}, e, g, g^{x}, g^{y}, g^{z}$, where $\left(\mathbb{G}_{1}, \mathbb{G}_{2}, e\right)$ is the output of $\mathcal{I G}$ for a sufficiently large security parameter $K, g$ is a random generator of $\mathbb{G}_{1}$, and $x$, $y, z$ are random elements of $\mathbb{Z}_{q}^{*}$. The BDH assumption is that $A d v_{I \mathcal{G}}(\mathcal{B})$ is negligible for any efficient $\mathcal{B}$.

\section{An Efficient Privacy Preserving Keyword Search Scheme}

\section{A. Definitions}

Suppose a user $U$ is about to store an encrypted email with keywords $W_{1}, \ldots, W_{k}$ on a service provider $S$, where $k \in \mathbb{Z}^{+}$. Keywords may be words in headline or accepted date, and $k$ is relatively small. $U$ sends the following message to $S$ :

$M S G_{U 2 S}=\left[E_{1}\left(U_{p u b}, S_{p u b}, m\right), E_{2}\left(U_{p u b}, W_{1}\right), \ldots, E_{2}\left(U_{p u b}, W_{k}\right)\right]$ where $U_{p u b}$ is $U$ 's public key, $S_{p u b}$ is $S$ 's public key, and $m$ is the email message body. $E_{1}$ and $E_{2}$ are public key encryption algorithms. $U$ encrypts the email message body using his own public key $U_{p u b}$, the service provider's public key $S_{p u b}$, and $E_{1}$. And then $U$ encrypts keywords $W_{1}, \ldots, W_{k}$ using his own public key $U_{p u b}$ and $E_{2}$. Finally, $U$ appends to the encrypted email message body with all the encrypted keywords and sends $M S G_{U 2 S}$ to $S$. 
Our goal is to enable $U$ to send a trapdoor $T_{W}$ for a certain keyword $W$ which is encrypted under his private key to $S$, which will enable $S$ to find out all emails containing the keyword $W$, but learns nothing else. $S$ then participates in the partial decipherment to calculate an intermediate result of the decipherment using its private key before returning the relevant encrypted emails back. We formally define the efficient privacy preserving keyword search scheme (EPPKS for short) below. EPPKS supports multiple keywords search on the encrypted data. For the sake of illustration, we only show a single keyword search case in this paper.

Definition 3.1 (EPPKS): EPPKS consists of seven randomized polynomial time algorithms as follows:

1) Keygen: takes a sufficiently large security parameter $K_{1}$ as an input, and produces a public/private key pair $\left(U_{p u b}, U_{\text {priv }}\right)$ for a user. We write Keygen $\left(K_{1}\right)=$ $\left(U_{\text {pub }}, U_{\text {priv }}\right)$. Let $K_{2}$ be a sufficiently large security parameter, we write Keygen $\left(K_{2}\right)=\left(S_{p u b}, S_{p r i v}\right)$ for a service provider.

2) EMBEnc: is a public key encryption algorithm that takes two public keys $U_{p u b}$ and $S_{p u b}$, and a message $m \in M$ as inputs, and produces $m$ 's cipher-text $C_{m} \in C_{M}$. We write EMBEnc $\left(U_{p u b}, S_{p u b}, m\right)=C_{m}$.

3) KWEnc: is a public key encryption algorithm that takes a public key $U_{p u b}$, and a keyword $W_{i} \in W\left(i \in \mathbb{Z}^{+}\right)$as inputs, and produces $W_{i}$ 's cipher-text $C_{W_{i}} \in C_{W}$. We write $K W E n c\left(U_{p u b}, W_{i}\right)=C_{W_{i}}$.

4) TCompute: takes a private key $U_{\text {priv }}$ and a keyword $W_{j}$ $\left(j \in \mathbb{Z}^{+}\right)$as inputs, and produces $W_{j}$ 's trapdoor $T_{W_{j}}$. We write TCompute $\left(U_{\text {priv }}, W_{j}\right)=T_{W_{j}}$.

5) Test: takes a public key $U_{p u b}$, an encrypted keyword $C_{W_{i}}$, and a trapdoor $T_{W_{j}}$ as inputs, and outputs 1 or 0. We write Test $\left(U_{p u b}, C_{W_{i}}, T_{W_{j}}\right)=1$ if $W_{i}=W_{j}$, and 0 otherwise.

6) Decrypt: takes a private key $S_{\text {priv }}$, a public key $U_{p u b}$, and a cipher-text $C_{m}$ as inputs, and outputs an intermediate result $C_{\rho}$. We write Decrypt $\left(S_{\text {priv }}, U_{p u b}, C_{m}\right)=C_{\rho}$.

7) Recovery: takes a private key $U_{p r i v}$, a cipher-text $C_{m}$, and an intermediate result $C_{\rho}$ as inputs, and outputs the plain-text $m$. We write Recovery $\left(U_{\text {priv }}, C_{m}, C_{\rho}\right)=m$.

The user and the service provider run Keygen to generate their public/private key pairs respectively. Given $U_{p u b}$ and $S_{p u b}, U$ runs EMBEnc to encrypt an email message body. Given $U_{p u b}, U$ runs KWEnc to encrypt keywords respectively. When $U$ wants to retrieve emails containing keyword $W_{j}\left(j \in \mathbb{Z}^{+}\right)$, he runs TCompute to generate $W_{j}$ 's trapdoor $T_{W_{j}}$ and sends it to $S . S$ runs Test to determine whether a given email contains keyword $W_{j}$ specified by $U . S$ runs Decrypt to calculate an intermediate result $C_{\rho}$ of the decipherment, and returns back $C_{\rho}$ along with the encrypted emails. Given a cipher-text and $C_{\rho}, U$ runs Recovery to recover the plain-text.

\section{B. Semantic Security of the EPPKS Scheme}

In this section, we define security for the EPPKS scheme in the sense of semantic security. Semantic security captures our intuition that given a cipher-text the adversary learns nothing about the corresponding plain-text, thus we also say that a semantically secure scheme is IND-CPA secure [8]. According to the definition of EPPKS, it consists of two public key encryption algorithms, i.e., KWEnc and EMBEnc. Therefore, we first define semantic security for EMBEnc and KWEnc, and then give our definition of a semantically secure EPPKS scheme.

Definition 3.2 (Semantic Security of KWEnc): Given a public key encryption algorithm KWEnc which encrypts keywords using $U_{p u b}$, let $\mathcal{A}_{1}$ be a polynomial time IND-CPA adversary that can adaptively ask for the trapdoor $T_{W_{i}}$ for any keyword $W_{i} \in W$ of its choice. $\mathcal{A}_{1}$ first chooses two keywords $W_{0}$ and $W_{1}$, which are not to be asked for trapdoors previously, and sends them to KWEnc. And then KWEnc picks a random element $b_{1} \in\{0,1\}$ and gives $\mathcal{A}_{1}$ the cipher-text $C_{W_{b_{1}}}=K W E n c\left(U_{p u b}, W_{b_{1}}\right)$. Finally, $\mathcal{A}_{1}$ outputs a guess $b_{1}^{\prime} \in\{0,1\}$ for $b_{1}$. We define the advantage of $\mathcal{A}_{1}$ in breaking KWEnc as $A d v_{\mathcal{A}_{1}}(k)=\left|\operatorname{Pr}\left[b_{1}=b_{1}^{\prime}\right]-\frac{1}{2}\right|$. We say that KWEnc is semantically secure if for any polynomial time adversary $\mathcal{A}_{1}$, the function $A d v_{\mathcal{A}_{1}}(k)$ is negligible.

Definition 3.3 (Semantic Security of EMBEnc): Given a public key encryption algorithm EMBEnc which encrypts the email message body using $U_{p u b}$ and $S_{p u b}$, let $\mathcal{A}_{2}$ be a polynomial time IND-CPA adversary that can adaptively ask for the cipher-text for any message $m_{i} \in M$ of its choice. $\mathcal{A}_{2}$ first chooses two messages $m_{0}$ and $m_{1}$, which are not to be asked for the cipher-text previously, and sends them to EMBEnc. And then EMBEnc picks a random $b_{2} \in\{0,1\}$ and gives $\mathcal{A}_{2}$ the cipher-text $C_{m_{b_{2}}}=\operatorname{EMBEnc}\left(U_{p u b}, S_{p u b}, m_{b_{2}}\right)$. Finally, $\mathcal{A}_{2}$ outputs a guess $b_{2}^{\prime} \in\{0,1\}$ for $b_{2}$. We define the advantage of $\mathcal{A}_{2}$ in breaking EMBEnc as $A d v_{\mathcal{H}_{2}}(k)=\left|\operatorname{Pr}\left[b_{2}=b_{2}^{\prime}\right]-\frac{1}{2}\right|$. We say that EMBEnc is semantically secure if for any polynomial time adversary $\mathcal{A}_{2}$, the function $A d v_{\mathcal{H}_{2}}(k)$ is negligible.

Definition 3.4 (Semantic Security of EPPKS): Given an EPPKS scheme consisting of KWEnc and EMBEnc, it takes a security parameter $K$ as input and runs the key generation algorithm Keygen to generate the public/private key pairs $\left(U_{p u b}, U_{p r i v}\right)$ and $\left(S_{p u b}, S_{p r i v}\right)$. Given an adversary $\mathcal{A}$ consisting of two polynomial time algorithms $\mathcal{A}_{1}$ and $\mathcal{A}_{2}, \mathcal{A}_{1}$ initiates attacks on KWEnc and $\mathcal{A}_{2}$ initiates attacks on EMBEnc. We say that the EPPKS Scheme is semantically secure if for any adversary $\mathcal{A}$, the function $A d v_{\mathcal{A}}(k)=A d v_{\mathcal{H}_{1}}(k)+A d v_{\mathcal{F}_{2}}(k)$ is negligible.

\section{Construction}

\section{A. Construction Based on Bilinear Maps}

Boneh et al [8] use bilinear maps on elliptic curves to build an efficient identity-based encryption (IBE) system. We construct EPPKS and prove that it is semantically secure, which closely follows that in Boneh et al [8]. Our construction is based on bilinear maps. The security of this scheme is based on the BDH assumption. Let IG be some BDH parameter generator. We present our scheme by describing the following seven algorithms. 
1) Keygen: Given a sufficiently large security parameter $K \in \mathbb{Z}^{+}$, it runs $I \mathcal{G}$ to generate a prime $q$, two groups $\mathbb{G}_{1}$ and $\mathbb{G}_{2}$ of prime order $q$, and a bilinear map e: $\mathbb{G}_{1} \times \mathbb{G}_{1} \rightarrow \mathbb{G}_{2}$, where $g$ is a generator of $\mathbb{G}_{1}$. Then it chooses two hash functions $H_{1}, H_{3}:\{0,1\}^{*} \rightarrow \mathbb{G}_{1}^{*}$, a hash function $H_{2}: \mathbb{G}_{2} \rightarrow\{0,1\}^{\log ^{q}}$, and a hash function $H_{4}$ : $\mathbb{G}_{2} \rightarrow\{0,1\}^{n}$ for some $n$, where $H_{1}, H_{2}, H_{3}$, and $H_{4}$ are random oracles. Finally, it picks two random elements $x, y \in \mathbb{Z}_{q}^{*}$ and computes $g^{x}$ and $g^{y}$. The plain-text space includes $M \in\{0,1\}^{n}$ and $W \in\{0,1\}^{*}$. The cipher-text space includes $C_{M}=\mathbb{G}_{1}^{*} \times\{0,1\}^{n}$ and $C_{W} \in \mathbb{G}_{2}$. The user's public key is $g^{x}$ with the corresponding private key $x$. The service provider's public key is $g^{y}$ with the corresponding private key $y$.

2) EMBEnc: To encrypt the email message body $m$ under a user's public key $g^{x}$ and a service provider's public key $g^{y}$, it picks a random element $r \in \mathbb{Z}_{q}^{*}$ and a random element $\rho \in\{0,1\}^{n}$, computes $u_{1}=g^{r}$, $u_{2}=\rho \oplus H_{4}\left(e\left(g^{x}, g^{y}\right)^{r}\right), u_{3}=m \oplus H_{4}\left(e\left(H_{3}(\rho), g^{x}\right)^{r}\right)$, and sets the cipher-text $C_{m}=\left(u_{1}, u_{2}, u_{3}\right)$.

3) KWEnc: To encrypt $m$ 's keywords $W_{1}, \ldots, W_{k}(k \in$ $\mathbb{Z}^{+}$) under a user's public key $g^{x}$, it computes $H_{2}\left(e\left(g^{x}, H_{1}\left(W_{i}\right)\right)^{r}\right)$, where $W_{i} \in\left\{W_{1}, \ldots, W_{k}\right\}$, sets the cipher-text $C_{W_{i}}=H_{2}\left(e\left(g^{x}, H_{1}\left(W_{i}\right)\right)^{r}\right)$, and sends the following message to the service provider:

$$
M S G_{U 2 S}^{\prime}=\left[C_{m}, C_{W_{1}}, \ldots, C_{W_{k}}\right]
$$

4) TCompute: To retrieve only emails containing keyword $W_{j}\left(j \in \mathbb{Z}^{+}\right)$, it computes the trapdoor $T_{W_{j}}=H_{1}\left(W_{j}\right)^{x} \in$ $\mathbb{G}_{1}$ under a user's private key $x$, and sends it to the service provider.

5) Test: To determine whether a given email contains keyword $W_{j}$, it tests whether $C_{W_{i}}=H_{2}\left(e\left(u_{1}, T_{W_{j}}\right)\right)=$ $H_{2}\left(e\left(g^{r}, T_{W_{j}}\right)\right)$. If so, Test $\left(U_{p u b}, C_{W_{i}}, T_{W_{j}}\right)$ outputs 1 . Otherwise, it outputs 0 . Note that: If $W_{i}=W_{j}$, then $\left.C_{W_{i}}=H_{2}\left(e\left(g^{x}, H_{1}\left(W_{i}\right)\right)^{r}\right)=H_{2}\left(g^{r}, H_{1}\left(W_{j}\right)^{x}\right)\right)=$ $H_{2}\left(e\left(g^{r}, T_{W_{j}}\right)\right)=H_{2}\left(e\left(u_{1}, T_{W_{j}}\right)\right)$ as required.

6) Decrypt: To get an intermediate result of the partial decipherment, it calculates $\rho$, computes $C_{\rho}=$ $e\left(H_{3}(\rho), u_{1}\right)=e\left(H_{3}(\rho), g^{r}\right)$, and sends the following results to the user:

$$
M S G_{S 2 U}=\left[C_{m}, C_{W_{1}}, \ldots, C_{W_{k}}, C_{\rho}\right]
$$

Note that: $\rho=u_{2} \oplus H_{4}\left(e\left(g^{x}, g^{y}\right)^{r}\right)=u_{2} \oplus H_{4}\left(e\left(g^{x}, g^{r}\right)^{y}\right)$. Therefore, it could calculate $\rho$ using a service provider's private key $y$.

7) Recovery: Given the cipher-text $C_{m}=\left(u_{1}, u_{2}, u_{3}\right)$ and $C_{\rho}$, it computes $m=u_{3} \oplus H_{4}\left(\left(C_{\rho}\right)^{x}\right)$ to recover the message $m$. Note that: $m=u_{3} \oplus H_{4}\left(e\left(H_{3}(\rho), g^{x}\right)^{r}\right)=$ $u_{3} \oplus H_{4}\left(e\left(H_{3}(\rho), g^{r}\right)^{x}\right)=u_{3} \oplus H_{4}\left(\left(C_{\rho}\right)^{x}\right)$.

For the sake of reducing the computational overhead and increasing the search speed, a service provider could calculate $\rho$ as soon as it receives $M S G_{U 2 S}^{\prime}$, and stores the message as follows:

$$
M S G_{\text {Stored@S }}=\left[C_{m}, C_{W_{1}}, \ldots, C_{W_{k}}, \rho\right]
$$

According to the PEKS scheme proposed in Boneh et al [7], a user encrypts the email message body using a standard public key system, and a service provider simply returns the relevant emails back after finishing the search. Our EPPKS scheme enables a service provider to participate in the partial decipherment to get an intermediate result of the decipherment before returning back the search results, but has no ability to recover the plain-text, which will reduce the computational overhead of the client greatly. Now, we show how to implement these improvements. In the next section, we will give a detailed proof of EPPKS.

According to EPPKS, a service provider is able to calculate $\rho$ using its private key $y$. Suppose the service provider knows $H_{3}(\rho)=g^{a} \in \mathbb{G}_{1}$ where $a$ is a random element in $\mathbb{Z}_{q}^{*}$, $u_{1}=g^{r} \in \mathbb{G}_{1}$ where $r \in \mathbb{Z}_{q}^{*}$ is a random element chosen by the user, and the user's public key $g^{x} \in \mathbb{G}_{1}$ where $x \in \mathbb{Z}_{q}^{*}$ is the user's private key, it couldn't calculate $e(g, g)^{a r x}$, assuming the $\mathrm{BDH}$ problem is hard. In other words, a service provider needs to compute $m=u_{3} \oplus H_{4}\left(e\left(H_{3}(\rho), g^{x}\right)^{r}\right)=u_{3} \oplus H_{4}\left(e(g, g)^{a r x}\right)$ to recover the plain-text, which corresponds to computing the BDH problem. Therefore, the service provider, seeing only a random value $\rho$ and calculating an intermediate result of the decipherment, has no idea what the plain-text is. Furthermore, an outer attacker couldn't calculate $\rho$ if he doesn't capture any private key. Suppose an outer attacker knows $u_{1}=g^{r}$ by intercepting $M S G_{U 2 S}^{\prime}$, the user's public key $g^{x} \in \mathbb{G}_{1}$, and the service provider's public key $g^{y} \in \mathbb{G}_{1}$, it couldn't calculate $e(g, g)^{x y r}$, assuming the BDH problem is hard. In other words, an outer attacker needs to compute $\rho=u_{2} \oplus H_{4}\left(e\left(g^{x}, g^{y}\right)^{r}\right)=u_{2} \oplus H_{4}\left(e(g, g)^{x y r}\right)$ to recover $\rho$, which corresponds to computing the BDH problem.

\section{B. Security}

In this section, we study the security of the proposed EPPKS scheme. The following theorem shows that EPPKS is semantically secure if the BDH problem is assumed to be hard.

Theorem 4.1: Suppose the hash functions $\mathrm{H}_{1}, \mathrm{H}_{2}, \mathrm{H}_{3}$, and $H_{4}$ are random oracles. Then EPPKS is semantically secure assuming the BDH problem is hard. Let $\mathcal{A}$ be an IND-CPA adversary consisting of two polynomial time algorithms $\mathcal{A}_{1}$ and $\mathcal{A}_{2}$. Let $\mathcal{A}_{1}$ be an IND-CPA adversary that has the advantage $\epsilon_{1}$ in breaking KWEnc. Suppose $\mathcal{A}_{1}$ makes $q_{T}>0$ trapdoor queries and $q_{H_{2}}>0$ hash queries to $H_{2}$. Let $\mathcal{A}_{2}$ be an IND-CPA adversary that has the advantage $\epsilon_{2}$ against EMBEnc. Suppose $\mathcal{A}_{2}$ makes $q_{H_{4}}>0$ hash function queries to $H_{4}$. Let $\mathcal{A}$ be an IND-CPA adversary that has the advantage $\epsilon=\epsilon_{1}+\epsilon_{2}$ against the EPPKS scheme. Then there is an algorithm $\mathcal{B}$ that solves the BDH problem with the advantage at least:

$$
A d \nu_{\mathcal{B}}(K) \geq 2 \epsilon_{1} /\left\{e \cdot q_{H_{2}} \cdot\left(1+q_{T}\right)\right\}+2 \epsilon_{2} / q_{H_{4}}
$$

Here $e \approx 2.71$ is the base of the natural logarithm. The running time of $\mathcal{B}$ is $O(\operatorname{time}(\mathcal{A}))$.

EPPKS includes two public key encryption algorithms, i.e., EMBEnc and KWEnc. Therefore, we prove Theorem 
4.1 in two steps. We first show that KWEnc is semantically secure if the BDH assumption holds.

Lemma 4.2: Let $H_{1}$ be a random oracle from $\{0,1\}^{*}$ to $\mathbb{G}_{1}^{*}$ and $H_{2}$ be a random oracle from $\mathbb{G}_{2}$ to $\{0,1\}^{\log ^{q}}$. Suppose $\mathcal{A}_{1}$ be an IND-CPA adversary that has the advantage $\epsilon_{1}$ in breaking KWEnc. Suppose $\mathcal{A}_{1}$ makes at most $q_{H_{2}}>0$ hash queries to $H_{2}$ and at most $q_{T}>0$ trapdoor queries. Then there is an algorithm $\mathcal{B}_{1}$ that solves the BDH problem with the advantage at least $\epsilon_{1}^{\prime}=2 \epsilon_{1} /\left\{e \cdot q_{H_{2}} \cdot\left(1+q_{T}\right)\right\}$, and a running time $O\left(\operatorname{time}\left(\mathcal{A}_{1}\right)\right)$.

Proof. Let $<q, \mathbb{G}_{1}, \mathbb{G}_{2}, e>$ be the BDH parameters, where $q$ is the prime order of $\mathbb{G}_{1}$ and $\mathbb{G}_{2}$. Choose a random generator $g \in \mathbb{G}_{1} . \mathcal{B}_{1}$ is given $v_{0}=g, v_{1}=g^{\alpha_{1}}, v_{2}=g^{\beta_{1}}, v_{3}=g^{\gamma_{1}} \in \mathbb{G}_{1}$ where $\alpha_{1}, \beta_{1}, \gamma_{1}$ are random elements in $\mathbb{Z}_{q}^{*}$. Its goal is to output $D_{1}=e(g, g)^{\alpha_{1} \beta_{1} \gamma_{1}} \in \mathbb{G}_{2}$. Let $D_{1}$ be the solution to the BDH problem. $\mathcal{B}_{1}$ finds $D_{1}$ by interacting with $\mathcal{A}_{1}$ as follows:

Keygen: $\mathcal{B}_{1}$ sends $\left(v_{0}, v_{1}\right)$ as the public key to $\mathcal{A}_{1}$.

$\mathbf{H}_{1}$-Queries: $\mathcal{B}_{1}$ maintains a list of tuples called $H_{1}$-List, in which each entry is a tuple of the form $<W_{j}, h_{j}, a_{j}, c_{j}>$. The list is initially empty. When $\mathcal{A}_{1}$ queries the random oracle $H_{1}$ at a point $W_{i} \in\{0,1\}^{*}, \mathcal{B}_{1}$ responds as follows:

1) If $W_{i}$ already appears on $H_{1}$-List in a tuple $<W_{i}, h_{i}, a_{i}, c_{i}>$, then $\mathcal{B}_{1}$ responds with $H_{1}\left(W_{i}\right)=h_{i} \in$ $\mathbb{G}_{1}^{*}$.

2) Otherwise, $\mathcal{B}_{1}$ generates a random coin $\in\{0,1\}$, so that $\operatorname{Pr}[$ coin $=0]=\delta$ for some $\delta$ that will be determined later.

3) $\mathcal{B}_{1}$ picks a random $a \in \mathbb{Z}_{q}^{*}$. If coin $=0, \mathcal{B}_{1}$ computes $h_{i}=v_{2} \cdot g^{a}=g^{\beta_{1}} \cdot g^{a} \in \mathbb{G}_{1}^{*}$. If coin $=1, \mathcal{B}_{1}$ computes $h_{i}=g^{a} \in \mathbb{G}_{1}^{*}$.

4) $\mathcal{B}_{1}$ adds the tuple $<W_{i}, h_{i}, a$, coin $>$ to $H_{1}$-List and responds to $\mathcal{A}_{1}$ with $H_{1}\left(W_{i}\right)=h_{i}$. Note that either way $h_{i}$ is uniform in $\mathbb{G}_{1}^{*}$ and is independent of $\mathcal{A}_{1}$ 's current view as required.

$\mathbf{H}_{2}$-Queries: $\mathcal{B}_{1}$ maintains a list of tuples called $H_{2}$-List, in which each entry is a tuple of the form $\left\langle t_{j}, v_{j}\right\rangle$. The list is initially empty. When $\mathcal{A}_{1}$ issues a query to $H_{2}, \mathcal{B}_{1}$ checks if $t_{i}$ is already on $H_{2}$-List in the form of $\left\langle t_{i}, v_{i}\right\rangle$. If so, $\mathcal{B}_{1}$ responds to $\mathcal{A}_{1}$ with $H_{2}\left(t_{i}\right)=v_{i}$. Otherwise, $\mathcal{B}_{1}$ picks a random string $v_{i} \in\{0,1\}^{\log ^{q}}$, adds the tuple $<t_{i}, v_{i}>$ to $H_{2}$-List, and responds to $\mathcal{A}_{1}$ with $H_{2}\left(t_{i}\right)=v_{i}$.

Phase 1: When $\mathcal{A}_{1}$ issues a query for the trapdoor of keyword $W_{i}, \mathcal{B}_{1}$ responds as follows:

1) $\mathcal{B}_{1}$ initiates $H_{1}$-Queries to obtain $h_{i} \in \mathbb{G}_{1}^{*}$, where $H_{1}\left(W_{i}\right)=h_{i}$. Let $<W_{i}, h_{i}, a_{i}, c_{i}>$ be the corresponding tuple on $H_{1}$-List. If $c_{i}=0$, then $\mathcal{B}_{1}$ reports a failure and terminates.

2) If $c_{i}=1$, then $H_{1}\left(W_{i}\right)=h_{i}=g^{a_{i}} \in \mathbb{G}_{1}^{*}$. We define $T_{W_{i}}=\left(v_{1}\right)^{a_{i}}=\left(g^{\alpha_{1}}\right)^{a_{i}}$. Note that $T_{W_{i}}=\left(g^{\alpha_{1}}\right)^{a_{i}}=\left(g^{a_{i}}\right)^{\alpha_{1}}=$ $H_{1}\left(W_{i}\right)^{\alpha_{1}}$. $\mathcal{B}_{1}$ gives $T_{W_{i}}$ to $\mathcal{A}_{1}$.

Challenge: Once $\mathcal{A}_{1}$ decides that Phase 1 is over, it outputs a pair of keywords $W_{0}$ and $W_{1}$ on which it wishes to be challenged. $\mathcal{B}_{1}$ responds as follows:

1) $\mathcal{B}_{1}$ initiates $H_{1}$-Queries twice to obtain $h_{0}$ and $h_{1} \in \mathbb{G}_{1}^{*}$, where $H_{1}\left(W_{0}\right)=h_{0}$ and $H_{1}\left(W_{1}\right)=h_{1}$. If $c_{0}=1$ or $c_{1}=1$, then $\mathcal{B}_{1}$ reports a failure and terminates.

2) If both $c_{0}=0$ and $c_{1}=0, \mathcal{B}_{1}$ randomly picks a $b_{1} \in$ $\{0,1\}$.

3) $\mathcal{B}_{1}$ picks a random string $S_{1} \in\{0,1\}^{\log ^{q}}$, and gives the cipher-text $C_{1}=\left(v_{3}, S_{1}\right)$ to $\mathcal{A}_{1}$. Note that:

$\left.S_{1}=H_{2}\left(e\left(v_{1}, H_{1}\left(W_{b_{1}}\right)\right)^{\gamma_{1}}\right)=H_{2}\left(g^{\alpha_{1}}, H_{1}\left(W_{b_{1}}\right)\right)^{\gamma_{1}}\right)$

$=H_{2}\left(e\left(g^{\alpha_{1}}, g^{\beta_{1}} \cdot g^{a_{b}}\right)^{\gamma_{1}}\right)=H_{2}\left(e(g, g)^{\alpha_{1} \gamma_{1}\left(\beta_{1}+a_{b}\right)}\right)$

Hence, $C_{1}$ is a valid cipher-text for $W_{b_{1}}$ as required.

Phase 2. $\mathcal{A}_{1}$ can continue issuing more trapdoor queries for keyword $W_{i}$, where the only restriction is that $W_{i} \neq W_{0}$ and $W_{i} \neq W_{1} . \mathcal{B}_{1}$ responds as in Phase 1.

Guess: $\mathcal{A}_{1}$ outputs its guess $b_{1}^{\prime} \in\{0,1\}$ for $b_{1}$. $\mathcal{B}_{1}$ picks a random pair $\left\langle t_{i}, v_{i}>\right.$ from $H_{2}$-List and outputs $t_{i}$ as the solution to $D_{1}$.

To complete the proof of Lemma 4.2, we now show that $\mathcal{B}_{1}$ correctly outputs $D_{1}$ with the probability at least $\epsilon_{1}^{\prime}=2 \epsilon_{1} /\left\{e \cdot q_{H_{2}} \cdot\left(1+q_{T}\right)\right\}$. In the first place, we calculate the probability that $\mathcal{B}_{1}$ does not abort during the above process. Suppose $\mathcal{A}_{1}$ makes a total of $q_{T}$ trapdoor queries. Then the probability $\mathcal{B}_{1}$ does not abort in Phase $\mathbf{1}$ or $\mathbf{2}$ is $\delta^{q_{T}}$. And the probability that it does not abort during the challenge step is $1-\delta$. Therefore, the probability that $\mathcal{B}_{1}$ does not abort during the whole process is $\delta^{q_{T}} \cdot(1-\delta)$. This value is maximized at $\delta_{\text {opt }}=1-1 /\left(q_{T}+1\right)$. Using $\delta_{\text {opt }}$, the probability that $\mathcal{B}_{1}$ does not abort is at least $1 / e\left(1+q_{T}\right)$. In the second place, we calculate the probability that $\mathcal{B}_{1}$ outputs the correct result in case that $\mathcal{B}_{1}$ does not abort. Let $Q_{1}$ be the event that $\mathcal{A}_{1}$ issues a query for $v$. If $\neg Q_{1}$, we know that the decryption of the cipher-text is independent of $\mathcal{A}_{1}$ 's view. Let $\operatorname{Pr}\left[b_{1}=b_{1}^{\prime}\right]$ be the probability that $\mathcal{A}_{1}$ outputs the correct result, therefore in the real attack $\operatorname{Pr}\left[b_{1}=b_{1}^{\prime} \mid \neg Q_{1}\right]=\frac{1}{2}$. Since $\mathcal{A}_{1}$ has the advantage $\epsilon_{1},\left|\operatorname{Pr}\left[b_{1}=b_{1}^{\prime} \mid \neg Q_{1}\right]-\frac{1}{2}\right| \geq \epsilon_{1}$. According to the following formulae, we know $\operatorname{Pr}\left[Q_{1}\right] \geq 2 \epsilon_{1}$.

$$
\begin{aligned}
\operatorname{Pr}\left[b_{1}=b_{1}^{\prime}\right] & =\operatorname{Pr}\left[b_{1}=b_{1}^{\prime} \mid \neg Q_{1}\right] \operatorname{Pr}\left[\neg Q_{1}\right] \\
& +\operatorname{Pr}\left[b_{1}=b_{1}^{\prime} \mid Q_{1}\right] \operatorname{Pr}\left[Q_{1}\right] \\
& \leq \frac{1}{2} \operatorname{Pr}\left[\neg Q_{1}\right]+\operatorname{Pr}\left[Q_{1}\right] \\
& =\frac{1}{2}+\frac{1}{2} \operatorname{Pr}\left[Q_{1}\right] \\
\operatorname{Pr}\left[b_{1}=b_{1}^{\prime}\right] & \geq \operatorname{Pr}\left[b_{1}=b_{1}^{\prime} \mid \neg Q_{1}\right] \operatorname{Pr}\left[\neg Q_{1}\right] \\
& =\frac{1}{2} \operatorname{Pr}\left[\neg Q_{1}\right] \\
& =\frac{1}{2}-\frac{1}{2} \operatorname{Pr}\left[Q_{1}\right]
\end{aligned}
$$

Therefore, we have that $\operatorname{Pr}\left[Q_{1}\right] \geq 2 \epsilon_{1}$ in the real attack. Now we know that $\mathcal{A}_{1}$ will issue a query for $v$ with the probability at least $2 \epsilon_{1}$. That is to say, the probability that $v$ appears in some pair on $H_{2}$-List is at least $2 \epsilon_{1} . \mathcal{B}_{1}$ will choose the correct pair with the probability at least $1 / q_{H_{2}}$ and thus $\mathcal{B}_{1}$ produces the correct answer with the probability at least $2 \epsilon_{1} / q_{H_{2}}$. Since $\mathcal{B}_{1}$ does not abort with the probability at least $1 / e\left(1+q_{T}\right)$, we see that $\mathcal{B}_{1}$ 's success probability is at least $\epsilon_{1}^{\prime}=2 \epsilon_{1} /\left\{e \cdot q_{H_{2}} \cdot\left(1+q_{T}\right)\right\}$ as required.

Next, we show that EMBEnc is a semantically secure public key encryption if the BDH assumption holds. It is worth noticing that the outer attackers couldn't calculate $\rho$ if the BDH assumption holds. Without loss of generality, we suppose that an IND-CPA adversary $\mathcal{A}_{2}$ has already known $\rho$ and could issue $H_{3}$ queries at any time.

Lemma 4.3: Let $H_{3}$ be a random oracle from $\{0,1\}^{*}$ to 
$\mathbb{G}_{1}^{*}$ and $H_{4}$ be a random oracle from $\mathbb{G}_{2}$ to $\{0,1\}^{n}$. Let $\mathcal{A}_{2}$ be an IND-CPA adversary that has the advantage $\epsilon_{2}$ against EMBEnc. Suppose $\mathcal{A}_{2}$ makes $q_{H_{4}}>0$ hash function queries to $H_{4}$. Then there is an algorithm $\mathcal{B}_{2}$ that solves the $\mathrm{BDH}$ problem with the advantage at least $\epsilon_{2}^{\prime}=2 \epsilon_{2} / q_{H_{4}}$ and a running time $O\left(\operatorname{time}\left(\mathcal{A}_{2}\right)\right)$.

Proof. $\mathcal{B}_{2}$ is given $\rho \in\{0,1\}^{n}, \mu_{0}=g, \mu_{1}=g^{\alpha_{2}}, \mu_{2}=g^{\beta_{2}}$, $\mu_{3}=g^{\gamma_{2}} \in \mathbb{G}_{1}$, where $\alpha_{2}, \beta_{2}, \gamma_{2}$ are random elements in $\mathbb{Z}_{q}^{*}$. Its goal is to output $D_{2}=e(g, g)^{\alpha_{2} \beta_{2} \gamma_{2}} \in \mathbb{G}_{2}$. Let $D_{2}$ be the solution to the BDH problem. $\mathcal{B}_{2}$ finds $D_{2}$ by interacting with $\mathcal{A}_{2}$ as follows:

Keygen: $\mathcal{B}_{2}$ sends $\left(\mu_{0}, \mu_{1}\right)$ as the public key to $\mathcal{A}_{2}$.

$\mathbf{H}_{3}$-Queries: $\mathcal{B}_{2}$ maintains a list of tuples called $H_{3}$-List, in which each entry is a tuple of the form $\left\langle\rho_{j}, f_{j}\right\rangle$. The list is initially empty. When $\mathcal{A}_{2}$ issues a query to $H_{3}, \mathcal{B}_{2}$ checks if $\rho_{i}$ is already on $\mathrm{H}_{3}$-List in the form of $\left\langle\rho_{i}, f_{i}\right\rangle$. If so, $\mathcal{B}_{2}$ responds to $\mathcal{A}_{2}$ with $H_{3}\left(\rho_{i}\right)=f_{i}$. Otherwise, $\mathcal{B}_{2}$ picks a random $d \in \mathbb{Z}_{q}^{*}$, computes $f_{i}=\mu_{2} \cdot g^{d}=g^{\beta_{2}} \cdot g^{d} \in \mathbb{G}_{1}^{*}$, adds the tuple $<\rho_{i}, f_{i}>$ to $H_{3}$-List, and responds to $\mathcal{A}_{2}$ with $H_{3}\left(\rho_{i}\right)=f_{i}$.

$\mathbf{H}_{4}$-Queries: $\mathcal{B}_{2}$ maintains a list of tuples called $H_{4}$-List, in which each entry is a tuple of the form $\left\langle r_{j}, l_{j}\right\rangle$. The list is initially empty. When $\mathcal{A}_{2}$ issues a query to $H_{4}, \mathcal{B}_{2}$ checks if $r_{i}$ is already on $H_{4}$-List in the form of $\left\langle r_{i}, l_{i}\right\rangle$. If so, $\mathcal{B}_{2}$ responds to $\mathcal{A}_{2}$ with $H_{4}\left(r_{i}\right)=l_{i}$. Otherwise, $\mathcal{B}_{2}$ picks a random string $l_{i} \in\{0,1\}^{n}$, adds the tuple $<r_{i}, l_{i}>$ to $H_{4}$-List, and responds to $\mathcal{A}_{2}$ with $H_{4}\left(r_{i}\right)=l_{i}$.

Challenge. $\mathcal{A}_{2}$ outputs two messages $m_{0}$ and $m_{1}$ on which it wishes to be challenged. $\mathcal{B}_{2}$ randomly picks $b_{2} \in\{0,1\}$ and a random string $S_{2} \in\{0,1\}^{n}$, and gives the cipher-text $C_{2}=\left(\mu_{3}, S_{2}\right)$ to $\mathcal{A}_{2}$. Note that the decryption of the ciphertext is:

$m_{b_{2}}=S_{2} \oplus H_{4}\left(e\left(H_{3}(\rho), \mu_{1}\right)^{\gamma_{2}}\right)=S_{2} \oplus H_{4}\left(e\left(H_{3}(\rho), g^{\alpha_{2}}\right)^{\gamma_{2}}\right)$

$=S_{2} \oplus H_{4}\left(e\left(g^{\beta_{2}} \cdot g^{d}, g^{\alpha_{2}}\right)^{\gamma_{2}}\right)=S_{2} \oplus H_{4}\left(e(g, g)^{\alpha_{2} \gamma_{2}\left(\beta_{2}+d\right)}\right.$

Hence, $C_{2}$ is a valid cipher-text for $m_{b_{2}}$ as required.

Guess: $\mathcal{A}_{2}$ outputs its guess $b_{2}^{\prime} \in\{0,1\}$ for $b_{2}$. $\mathcal{B}_{2}$ picks a random pair $<r_{i}, l_{i}>$ from $H_{4}$-List and outputs $r_{i}$ as the solution to the given instance of BDH.

Let $Q_{2}$ be the event that $\mathcal{A}_{2}$ issues a query for $l$. From proof of Lemma 4.2, we know that $\operatorname{Pr}\left[Q_{2}\right] \geq 2 \epsilon_{2}$. That is to say, $\mathcal{A}_{2}$ will issue a query for $l$ with the probability at least $2 \epsilon_{2}$. $\mathcal{B}_{2}$ will choose the correct pair with the probability at least $1 / q_{H_{4}}$ and thus $\mathcal{B}_{2}$ produces the correct answer with the probability at least $\epsilon_{2}^{\prime}=2 \epsilon_{2} / q_{H_{4}}$ as required.

Proof of Theorem 4.1. The theorem follows directly from Lemma 4.2 and Lemma 4.3. It shows that an INDCPA adversary $\mathcal{A}$ on EPPKS with the advantage $\epsilon$ gives a BDH algorithm with the advantage at least $A d v_{\mathcal{B}} \geq$ $2 \epsilon_{1} / e \cdot q_{H_{2}} \cdot\left(1+q_{T}\right)+2 \epsilon_{2} / q_{H_{4}}$ as required.

\section{Conclusion}

Cloud computing is one of the current most important and promising technologies. A user could store his personal files in a cloud and retrieves them wherever and whenever he wants. For the sake of protecting the user data privacy and the user queries privacy, we propose an efficient privacy preserving keyword search scheme in cloud computing. It allows a service provider to participate in partial decipherment, thus the user could pay less computational overhead for decryption. Furthermore, it is a searchable encryption scheme, thus the service provider could search the encrypted files efficiently without leaking any information. By proof, it is semantically secure.

In many cases, the user might want the service provider not only to provide the storage service, but also to provide the computational service. Ideally, the service provider has the ability to output the right answers but knows nothing about the data of the user. In other words, the service provider needs to compute on the encrypted data, which is a big challenge for us. In our future work, we will dedicate to this research direction.

\section{ACKNOWLEDGMENTS}

This work is supported by the National Natural Science Foundation of China under Grant Nos. 90718034 and 60773013, the Program for New Century Excellent Talents in University (NCET-06-0686), and the Program for Changjiang Scholars and Innovative Research Team in University under Grant No. IRT0661.

\section{REFERENCES}

[1] A. Weiss. Computing in the Clouds. netWorker, 11(4):16-25, Dec. 2007.

[2] Gartner Identifies the Top 10 Strategic Technologies for 2009, http://www.gartner.com/it/page.jsp?id=777212/ [14 Oct 2008].

[3] Amazon Elastic Compute Cloud (EC2), http://www.amazon.com/ec2/ [18 Jul 2008].

[4] Google App Engine, http://appengine.google.com [18 Jul 2008].

[5] Microsoft Live Mesh, http://www.mesh.com [18 Jul 2008].

[6] H. Haclgiimfi, B. Iyer, C. Li, and S. Mehrotra. Executing SQL over Encrypted Data in Database-Service-Provider Model. Technical Report TR-DB-02-02, Database Research Group at University of California, Irvine, 2002.

[7] D. Boneh, G. Crescenzo, R. Ostrovsky, and G. Persiano. Public Key Encryption with Keyword Search. Proceedings of Eurocrypt 2004, Lecture Notes in Computer Science 3027, pp. 506-522.

[8] D. Boneh and M. Franklin. Identity Based Encryption from the Weil Pairing. SIAM J. of Computing, 32 (3): 586-615, 2003, also as an extended abstract in Crypto 2001.

[9] D. X. Song, D. Wagner and A. Perrig. Practical Techniques for Searches on Encrypted Data. Proceedings of the 2000 IEEE Symposium on Security and Privacy, pp. 44-55.

[10] A. K. Bennett, C. Grothoff, T. Horozov, and I. Patrascu. Efficient Sharing of Encrypted Data. Proceedings of ACISP 2002, Lecture Notes in Computer Science 2384, pp. 107-120.

[11] T. Diament, H. K. Lee, A. D. Keromytis and M. Yung. The Dual Receiver Cryptosystem and its Applications. Proceedings of the ACM CCS 2004, pp. 330-343.

[12] Y.-C. Chang and M. Mitzenmacher. Privacy Preserving Keyword Searches on Remote Encrypted Data. Proceedings of ACSN 2005, Lecture Notes in Computer Science 3531, pp. 442-455.

[13] M. Bellare, A. Boldyreva, A. Desai, and D. Pointcheval. Key-Privacy in Public-Key Encryption. Proceedings of Advances in Cryptology Asiacrypt 2001, Lecture Notes in Computer Science 2248, pp. 566-582.

[14] K. Ren, W. Lou, K. Kim, and R. Deng. A Novel Privacy Preserving Authentication and Access Control Scheme for Pervasive Computing Environment. IEEE Transactions on Vehicular Technology, 55(4):13731384, Jul. 2006.

[15] D. Boneh and B. Waters. Conjunctive, Subset, and Range Queries on Encrypted Data. Proceedings of TCC 2007, Lecture Notes in Computer Science 4392, pp. 535-554.

[16] E. Shi, J. Bethencourt, T-H. H. Chan, D. Song, and A. Perrig. MultiDimensional Range Query over Encrypted Data. Proceedings of IEEE Symposium on Security and Privacy 2007, pp. 350-364. 